\title{
Análise da Revisão Cochrane: Prescrição Não-médica \\ versus Prescrição Médica para a Gestão de Doenças \\ Agudas e Crónicas em Cuidados de Saúde Primários \\ e Secundários. Cochrane Database Syst Rev.
}

2016;11:CD011227.

\author{
Analysis of the Cochrane Review: Non-medical Prescribing \\ versus Medical Prescribing for Acute and Chronic Disease \\ Management in Primary and Secondary Care. Cochrane \\ Database Syst Rev. 2016;11:CD011227.
}

Gonçalo Silva DUARTE $1,2,3$, Ricardo Martins DELGADO ${ }^{3}$, João COSTA ${ }^{1,2,3}$, António VAZ-CARNEIRO $\triangle^{3,4}$

Acta Med Port 2017 Jan;30(1):7-11 - http://dx.doi.org/10.20344/amp.8652

\section{RESUMO}

Em países com diversos níveis socioeconómicos, o assegurar dos cuidados de saúde requer a consideração de uma variedade de estratégias, nas quais pode incluir-se a prescrição não-médica. O objetivo desta revisão foi averiguar se a prescrição por profissionais de saúde não-médicos está associada a resultados (outcomes) clínicos e de relevância para o doente comparáveis à prescrição médica. Revisão sistemática de acordo com a metodologia preconizada pela Cochrane. Foram comparados os outcomes em saúde quando a prescrição médica era realizada por um qualquer profissional de saúde não médico (que prescrevesse com um alto grau de autonomia) versus por um médico, no contexto dos cuidados de saúde primários e secundários, e em países de baixo, médio e alto nível socioeconómico. Foram incluídos para análise 46 ensaios clínicos de diferentes desenhos que avaliaram um total de 37337 indivíduos. A maioria dos estudos baseou-se na gestão da doença crónica em países de nível socioeconómico mais elevado, onde existe evidência que suporta que os outcomes são comparáveis para os marcadores substitutivos de controlo de doença relativos à hipertensão (evidência moderada), controlo de diabetes (evidência elevada) e hipercolesterolemia (evidência moderada), entre outros. A prescrição por enfermeiros e farmacêuticos com níveis variados de formação (pré-graduada, específica e pós-graduada) esteve também associada a outcomes globalmente comparáveis aos obtidos com a prescrição médica relativamente à adesão à medicação, eventos adversos, nível de satisfação global, qualidade de vida e utilização de recursos (hospitalizações, urgências hospitalares e consultas). Os prescritores não médicos frequentemente tiveram suporte médico disponível para facilitar um modelo de prática colaborativa. Com treino e suporte apropriados, a prescrição não-médica por enfermeiros e farmacêuticos é tão efetiva quanto a prescrição médica numa variedade de condições clínicas.

Palavras-chave: Doença Aguda/tratamento; Doença Crónica/tratamento; Prescrições de Medicamentos; Revisão Sistemática

\section{ABSTRACT}

In countries with diverse socioeconomic levels, in order to address the health care demands, the consideration of a wide array of strategies is needed; among these, non-medical prescription can be included. The objective of this revision was to ascertain whether non-medical prescription results in comparable clinical and patient-related outcomes to medical prescription. This systematic review was conducted in compliance with the standard Cochrane methodology. Clinical outcomes were compared between prescription carried out by non-medical health professionals and doctors, provided that the non-medical health professional prescribed with a high degree of autonomy, both in hospital and community practice, in countries with low, medium and high socioeconomic level. The review included 46 clinical trials, with different designs, with 37337 participants overall. Most studies were based on the management of chronic disease in higher income countries, where the evidence supports comparable outcomes for the markers of disease relative to high blood pressure (moderate-certainty of evidence), diabetes control (high-certainty of evidence); high cholesterol (moderatecertainty of evidence), among others. Prescription by pharmacists and nurses with different levels of undergraduate, specific and postgraduate education could provide comparable outcomes to medical prescription, specifically with regards to adherence to therapy, adverse events, overall satisfaction, quality of life, and resource utilisation (hospitalisations, visits to the emergency department, and consultations). Non-medical prescribers frequently had medical support available to facilitate a collaborative practice. With appropriate training and support, non-medical prescription by nurses and pharmacists can be as effective as when carried out by doctors.

Keywords: Acute Disease/therapy; Chronic Disease/therapy; Drug Prescriptions; Systematic Review

\footnotetext{
1. Laboratório de Farmacologia Clínica e Terapêutica. Faculdade de Medicina. Universidade de Lisboa. Lisboa. Portugal.

2. Clinical Pharmacology Unit. Instituto de Medicina Molecular. Lisboa. Portugal.

3. Centro de Estudos de Medicina Baseada na Evidência. Faculdade de Medicina. Universidade de Lisboa. Lisboa. Portugal.

4. Centro Colaborador Português da Rede Cochrane Iberoamericana. Lisboa. Portugal.

$\square$ Autor correspondente: António Vaz-Carneiro. avc@medicina.ulisboa.pt

Recebido: 09 de janeiro de 2017 - Aceite: 10 de janeiro de 2017 | Copyright @ Ordem dos Médicos 2017
} 


\section{QUESTÃO CLÍNICA}

Será que existe diferença quando a prescrição medicamentosa é feita por profissionais de saúde não-médicos comparativamente à prescrição por médicos, em termos de efeitos clínicos, resultados reportados pelos doentes ( $p a-$ tient-reported outcomes) e a utilização de recursos, tanto em doenças agudas e crónicas, e em cuidados de saúde primários e secundários.

\section{OBJECTIVOS}

Avaliarosefeitosclínicos(controlodadoença),ospatient-reported outcomes, e a utilização de recursos quando a prescrição medicamentosa é feita por não-médicos, em doenças agudas e crónicas, e em cuidados de saúde primários e secundários, quando comparado com a prescrição médica (cuidado habitual).

\section{METODOLOGIA}

Revisão sistemática de ensaios clínicos aleatorizados e controlados, ensaios clínicos aleatorizados em cluster, ensaios clínicos controlados com um período pré-pós e estudos de análise de séries temporais ininterruptas (com pelo menos três observações pré e pós a intervenção). As intervenções estudadas foram a prescrição medicamentosa feita por profissionais de saúde não médicos (enfermeiros, farmacêuticos, etc.) versus por médicos, em diferentes contextos de cuidados de saúde e condições clínicas. Para identificar os estudos relevantes foram pesquisadas as seguintes bases de dados e fontes de informação até julho de 2016: Cochrane Central Register of Controlled Trials; Cochrane Methodology Register, the Cochrane Library; Cochrane Database of Systematic Reviews; Database of Abstracts and Reviews of Effects; Health Technology Assessment Database, the Cochrane Library; NHS Economic Evaluation Database, the Cochrane Library; MEDLINE; EMBASE; PsycINFO; Cumulative Index to Nursing and Allied Health Literature (CINAHL); Open Grey; Grey Literature Report by the New York Academy of Medicine; Agency for Healthcare Research and Quality (AHRQ); ICTRP; e ClinicalTrials.gov. Dois investigadores independentes selecionaram os estudos, extraíram os dados e avaliaram a qualidade metodológica dos estudos. Os principais outcomes foram:

\section{Primários}

- Proporção de prescritores, tanto médicos como não-médicos, a aderir a normas de orientação clínicas;

- Adesão terapêutica;

- Proporção de doentes e itens apropriadamente prescritos e retirados;

- Satisfação dos doentes, quando medidos de forma devidamente validada;

- Tempo de espera para prescrição;

- Prescritores não-médicos que afetaram de forma negativa os resultados de saúde dos doentes através de erros na medicação, erros de prescrição, eventos adversos, diagnóstico ou terapêutica errada, aumento de hospitalizações, ou necessidade de cuidados médicos;

- Funções diárias e qualidade de vida relacionada com a saúde.

\section{Secundários}

- Satisfação profissional, desempenhos utilizados, autonomia diária e qualidade de vida;

- Tempo médico poupado pelos prescritores não-médicos;

- Volume e custo de prescrições por parte dos doentes, pagamentos directos, custos, taxas de interrupção da prescrição e respetivo custo;

- Utilização de recursos para providenciar intervenções subsequentes tais como hospitalizações, recorrer ao serviço de urgência, e consultas médicas.

\section{RESULTADOS}

Ao todo foram incluídos 46 estudos, com um total de 37337 participantes. A prescrição não-médica foi feita por enfermeiros em 26 estudos, e por farmacêuticos em 20 estudos. O grau de educação destes profissionais de saúde necessária para inclusão nos estudos foi variável de acordo com o país e local de estudo.

Nas análises de marcadores intermédios de doenças crónicas várias (pressão arterial sistólica, hemoglobina glicada, e colesterol de baixa densidade (LDL)) verificou-se em todas um melhor resultado no grupo de prescrição não-médica. Os autores consideraram existir evidência de moderada qualidade relativamente à maior redução de pressão arterial sistólica aos 12 meses (redução média comparada com a prescrição médica: $5,31 \mathrm{mmHg}$; IC 95\%: 4,16 a 6,46; 12 estudos, 4229 participantes) bem como relativa à maior redução de colesterol LDL (redução média comparada com a prescrição médica: 3,78 mg; IC 95\%: 2,52 a 5,23; sete estudos, 1469 participantes). A qualidade da evidência não foi avaliada como alta devido à presença de heterogeneidade considerável entre os estudos, particularmente em relação a intervenções multifacetadas e diferentes graus de autonomia na prescrição. Os autores consideraram existir evidência de alta qualidade relativamente à maior redução de hemoglobina glicada aos 12 meses (redução média comparada com a prescrição médica: 0,62; IC 95\% 0,38 a 0,85 ; seis estudos; 775 participantes).

Verificou-se uma pequena diferença no grau de adesão terapêutica, favorecendo o grupo de prescrição não-médica (aumento médio comparado com a prescrição médica: standardised mean difference 0,15; $95 \% \mathrm{Cl} 0,00$ a 0,30 ; quatro estudos, 700 participantes). A evidência que suporta este resultado foi classificada como de moderada qualidade, com o risco de viés de performance sendo a maior preocupação. Embora se tivesse verificado poucas diferenças em relação ao risco de eventos adversos, esta evidência foi classificada como sendo de baixa qualidade, uma vez que o espectro de eventos adversos pode não estar relacionado com a intervenção e os estudos que reportaram eventos adversos fizeram-no de forma seletiva. 
Os doentes ficaram geralmente satisfeitos com o cuidado dos prescritores não-médicos (14 estudos; 7514 participantes), no entanto a qualidade desta evidência é moderada. A análise de escalas de qualidade de vida relacionadas com a saúde (Short Form-12 e Short Form-36) favoreceu cuidados médicos na sua componente física (diferença média: 1,17; IC 95: 0,16 a 2,17) mas não na componente mental (diferença média: 0,58; IC $95 \% \mathrm{Cl}:-0,40$ a 1,55). Contudo, é possível que medições de qualidade de vida reflitam mais adequadamente o cuidado total do que componentes relacionados apenas com a prescrição, razão pela qual a evidência foi avaliada como sendo de qualidade moderada.
Houve uma grande variedade de medidas de utilização de recursos, com poucas diferenças entre os grupos em comparação relativamente à taxa de hospitalizações, visitas ao serviço de urgência, e consultas externas. A maioria dos estudos mostrou que os prescritores não-médicos prescreveram mais intervenções, intensificaram dosagens de fármacos mais frequentemente, e utilizaram uma maior variedade de fármacos, quando comparados com médicos.

Globalmente, as duas principais formas de viés foram de viés de performance e viés de seleção por não se conseguir ocultar o grupo de intervenção (allocation concealment).

Tabela 1 - Sumário de resultados, adotada do artigo original

\begin{tabular}{|c|c|c|c|c|c|c|}
\hline \multicolumn{7}{|c|}{$\begin{array}{l}\text { Pacientes ou população: pessoas com doenças aguda ou crónicas } \\
\text { Contexto: cuidados de saúde primários e secundários em países desenvolvidos e não-desenvolvidos } \\
\text { Intervenção: prescrição de intervenções terapêuticas feitas por não-médicos } \\
\text { Comparação: prescrição de intervenções terapêuticas feitas por médicos }\end{array}$} \\
\hline \multirow{3}{*}{ Outcome } & \multicolumn{2}{|c|}{ Riscos comparativos ilustrativos* (IC 95\%) } & \multirow{3}{*}{$\begin{array}{l}\text { Efeito relativo } \\
\text { (IC 95\%) }\end{array}$} & \multirow{3}{*}{$\begin{array}{l}\text { Número de } \\
\text { participantes } \\
\text { (estudos) }\end{array}$} & \multirow{3}{*}{$\begin{array}{l}\text { Qualidade da } \\
\text { evidência } \\
\text { (GRADE)** }\end{array}$} & \multirow{3}{*}{ Comentários } \\
\hline & Risco assumido & Risco correspondente & & & & \\
\hline & Prescrição médica & Prescrição não-médica & & & & \\
\hline $\begin{array}{l}\text { Pressão arterial } \\
\text { sistólica }(\mathrm{mmHg}) \\
\text { aos } 12 \text { meses }\end{array}$ & $\begin{array}{l}\text { A pressão arterial } \\
\text { sistólica média foi entre } \\
124 \mathrm{mmHg} \text { a } 149 \mathrm{mmHg}\end{array}$ & $\begin{array}{l}\text { A pressão arterial sistólica } \\
\text { média foi } 5,31 \mathrm{mmHg} \\
\text { mais baixa } \\
\text { (IC } 95 \% 4,16 \text { a } 6,46)\end{array}$ & - & $\begin{array}{c}4229 \\
\text { (12 estudos) }\end{array}$ & Moderada ${ }^{1,2,3}$ & $\begin{array}{l}\text { Redução média de } \\
5,91 \mathrm{mmHg} \\
\text { (IC } 95 \% 7,71 \text { a } 4,10 \text { ) }\end{array}$ \\
\hline $\begin{array}{l}\text { Hemoglobina glicada } \\
\text { (HbA1c, \%) } \\
\text { aos } 12 \text { meses }\end{array}$ & $\begin{array}{l}\text { A hemoglobina glicada } \\
\text { média foi entre } \\
-0,90 \% \text { a } 9,7 \%\end{array}$ & $\begin{array}{l}\text { A hemoglobina glicada } \\
\text { média foi } 0,62 \% \text { mais } \\
\text { baixa } \\
\text { (IC } 95 \% 0,38 \text { a } 0,85)\end{array}$ & - & $\begin{array}{c}775 \\
\text { (6 estudos) }\end{array}$ & Alta ${ }^{2,3}$ & $\begin{array}{l}\text { Redução média de } \\
0,62 \mathrm{HbA} 1 \mathrm{c} \\
\text { (IC } 95 \% 0,85 \text { a 0,38) }\end{array}$ \\
\hline $\begin{array}{l}\text { Colesterol LDL }(\mathrm{mg} / \mathrm{L}) \\
\text { aos } 12 \text { meses }\end{array}$ & $\begin{array}{l}\text { A redução de colesterol } \\
\text { LDL médio foi entre } \\
-4,68 \text { a } 61,44 \mathrm{mg} / \mathrm{L}\end{array}$ & $\begin{array}{l}\text { O colesterol LDL médio foi } \\
3,78 \mathrm{mg} / \mathrm{L} \text { mais baixa } \\
\text { (IC } 95 \% 2,52 \text { a } 5,23 \text { ) }\end{array}$ & - & $\begin{array}{c}1469 \\
\text { (7 estudos) }\end{array}$ & Moderada ${ }^{1,2,3}$ & $\begin{array}{l}\text { Redução média de } \\
5,41 \mathrm{mg} / \mathrm{L} \\
\text { (IC } 95 \% 0,36 \text { a 11,17) }\end{array}$ \\
\hline $\begin{array}{l}\text { Adesão terapêutica } \\
\text { aos } 6 \text { meses }\end{array}$ & $\begin{array}{l}\text { A adesão terapêutica } \\
\text { média foi de } 0,79\end{array}$ & $\begin{array}{l}\text { A adesão terapêutica } \\
\text { média foi } 0,15 \text { mais alta } \\
\text { (IC } 95 \% 0,00 \text { a 0,30) }\end{array}$ & - & $\begin{array}{c}700 \\
\text { (4 estudos) }\end{array}$ & Moderada 4,5 & - \\
\hline Satisfação de doentes & \multicolumn{2}{|c|}{$\begin{array}{l}\text { A maioria dos questionários não tinha uma fonte citada, } \\
\text { ou foram desenvolvidos localmente. Seis estudos } \\
\text { reportaram a satisfação dos prescritores e não dos } \\
\text { doentes. Um estudo reportou uma satisfação total de } \\
\text { doentes com a prescrição de um farmacêutico em } 85 \% \\
\text { dos participantes, embora } 9 \% \text { tenham dito que teriam } \\
\text { preferido ir ao seu médico de família }\end{array}$} & Não estimável & $\begin{array}{c}7514 \\
\text { (14 estudos) }\end{array}$ & Moderada 8,9 & - \\
\hline Eventos adversos & \multicolumn{2}{|c|}{$\begin{array}{l}\text { Houve uma pequena ou nenhuma diferença no risco de } \\
\text { eventos adversos entre os dois grupos em } 9 \text { estudos. } \\
\text { Dois estudos reportaram um risco superior no grupo de } \\
\text { prescrição médica. }\end{array}$} & Não estimável & $\begin{array}{c}18400 \\
\text { (18 estudos) }\end{array}$ & Baixa 6,7 & - \\
\hline $\begin{array}{l}\text { Qualidade de vida } \\
\text { relacionada com a } \\
\text { saúde }\end{array}$ & $\begin{array}{l}\text { A qualidade de vida } \\
\text { relacionada com a saúde } \\
\text { média foi de } 0\end{array}$ & $\begin{array}{l}\text { Componente físico } \\
1,17 \text { mais alto } \\
\text { (IC } 95 \% 0,16 \text { a } 2,17) \\
\text { Componente mental } \\
0,58 \text { mais alto } \\
\text { (IC } 95 \%-0,40 \text { a } 1,55)\end{array}$ & - & $\begin{array}{c}4631 \\
\text { (8 estudos) }\end{array}$ & Moderada ${ }^{10}$ & - \\
\hline
\end{tabular}

IC: Intervalo de confiança

* O risco assumido define-se como a mediana do risco dos grupos de controlo. O risco correspondente baseia-se no risco assumido no grupo de comparação e o efeito relativo da

intervenção.

** Sistema GRADE:

- Qualidade alta: Investigação ulterior é altamente improvável que altere a confiança na estimativa do efeito;

- Qualidade moderada: Investigação ulterior tem provável impacto na confiança na estimativa do efeito e pode mesmo alterá-la;

- Qualidade baixa: Investigação ulterior tem alta probabilidade de alterar o impacto da confiança na estimativa do efeito e de alterar a própria estimativa do efeito;

- Qualidade muito baixa: Grande incerteza na estimativa do resultado.

${ }^{1}$ Redução de um nível devido a inconsistência (heterogeneidade); ${ }^{2}$ Intervenções multifacetadas; ${ }^{3}$ Autonomia na prescrição variável; ${ }^{4}$ Redução de um nível devido a risco de viés elevado; ${ }^{5}$ Medidas de adesão reportadas de forma variável; ${ }^{6}$ Redução de um nível devido a variedade de eventos adversos, e por talvez não estar relacionado com a intervenção; ${ }^{7}$ Redução de um nível devido a reportagem seletiva de resultados, em particular os eventos adversos, que não foram reportados na maioria dos estudos; ${ }^{8}$ Redução de um nível devido a indirectness; ${ }^{9}$ Variabilidade em medidas de satisfação; ${ }^{10}$ Redução de um nível devido a indirectness. 


\section{CONCLUSÃO}

Os resultados desta revisão sistemática sugerem que a prescrição medicamentosa por prescritores não-médicos, exercendo com graus variáveis embora altos de autonomia, numa variedade de situações, é tao efetiva quanto a prescrição por médicos. Os resultados clínicos verificados para a pressão arterial sistólica, hemoglobina glicada, colesterol LDL, adesão terapêutica, satisfação do doente, e qualidade de vida em geral podem ser considerados equiparáveis.

É difícil determinar o exato impacto da prescrição não-médica comparada com a médica em relação a eventos adversos e utilização de recursos devido à inconsistência e variabilidade de como estes outcomes foram avaliados e descritos nos estudos.

\section{COMENTÁRIO}

A presente revisão sistemática aborda uma temática particularmente relevante, e não mais clarificada para a globalidade dos profissionais de saúde com funções assistenciais em Portugal.

Com a sub-especialização da classe médica, tem vindo a constatar-se que esta tendência se acompanha de aspectos positivos para as funções assistenciais mas, também, de aspectos negativos que devem ser endereçados. De entre os positivos, destacam-se o nível de cuidados que, globalmente, são actualmente providenciados aos doentes em cuidados secundários e terciários: das revascularizações percutâneas ao último agente biológico para o tratamento de uma neoplasia antes intratável, são poucos - ou mesmo inexistentes - os campos da medicina em que a acção concertada de todos os profissionais de saúde não tenha sido capaz de prolongar vidas que antes se encontravam inabalavelmente condicionadas.

No entanto, o nível de cuidados que o sistema mais ou menos integrado de profissionais de saúde providencia encontra-se ameaçado, frequentemente, por motivos de limitação na prestação de cuidados primários. $O$ aumento de recursos terapêuticos e a capacidade técnico-científica contrastam com a realidade presente de um número substancial de doentes que não se encontra convenientemente medicado para patologias como a hipertensão arterial, a diabetes mellitus e dislipidemia, em que a farmacopeia actual permite, com provas dadas, providenciar uma diminuição significativa do risco de eventos secundários e aumento da sobrevida das pessoas com estas patologias. Resumindo: tratamos o extremamente difícil, e podemos ficar aquém no que deveria ser fácil.

A obrigatoriedade do registo clínico informático - que acarreta vantagens óbvias, ao nível da comunicação entre os diferentes intervenientes na saúde - conjuga-se com uma realidade em que o ónus da burocracia ameaça reduzir a disponibilidade assistencial, essencial para a manutenção da saúde e a prevenção da doença.

Assume-se relevante explicitar, no entanto, que a so- bre-especialização médica se tem acompanhado de um aumento da especialização das demais classes de profissionais de saúde. Embora exista uma falta de disponibilidade organizacional em providenciar os cuidados primários necessários, existe também um enorme potencial que poderá - e deverá - ser considerado na tentativa de diminuir as limitações no acesso a cuidados de saúde. Nesta revisão sistemática, analisa-se o aspecto da prescrição não-médica de medicamentos.

A análise dos resultados desta revisão permite concluir que os resultados da prescrição medicamentosa por prescritores não-médicos e médicos parecem ser equiparáveis no controlo da pressão arterial sistólica, diabetes e dislipidemia, na adesão terapêutica, satisfação do doente, e qualidade de vida em geral. Em particular, a prescrição medicamentosa por enfermeiros e farmacêuticos com níveis variáveis de treino e educação e com um fácil apoio médico ao seu alcance está associada a resultados equiparáveis aos obtidos com a prescrição médica.

De facto, prescritores não-médicos em países desenvolvidos e não-desenvolvidos podem contribuir para reduzir o peso de doenças crónicas, ou em situações de falta de médicos ou falta de recursos de saúde, ao providenciar uma complementaridade importante à acção médica no momento da prescrição.

\section{IMPLICAÇÕES PARA A PRÁTICA CLÍNICA}

- Prescritores não-médicos exercem em múltiplas situações e com níveis variáveis, embora geralmente altos, de autonomia, sendo que os resultados obtidos parecem ser comparáveis na gestão de doenças crónicas e em cuidados de prevenção.

- Os resultados da prescrição medicamentos por prescritores não-médicos são equiparáveis em termos de controlo da pressão arterial sistólica, diabetes, colesterol LDL, adesão terapêutica, satisfação do doente, e qualidade de vida em geral.

- A baixa qualidade da evidência em estudos que reportam eventos adversos e utilização de recursos torna difícil a comparação entre prescritores não-médicos e médicos. Os aspectos da segurança medicamentosa são cada vez mais centrais e os estudos futuros devem avaliar especificamente o impacto da prescrição não médica neste aspecto.

- Os resultados obtidos com enfermeiros e farmacêuticos parecem ser equiparáveis, com níveis variáveis de treino e educação.

- Prescritores não-médicos frequentemente têm apoio médico ao seu alcance, se necessário, e nestas circunstâncias uma abordagem de colaboração aparenta ser o modelo de cuidados preferível. Os prescritores não-médicos em países desenvolvidos e não-desenvolvidos podem contribuir para reduzir o peso de doenças crónicas, ou em situações de escassez de médicos ou de recursos de saúde. 


\section{REFERÊNCIAS}

1. Weeks G, George J, Maclure K, Stewart D. Non-medical prescribing versus medical prescribing for acute and chronic disease management in primary and secondary care. Cochrane Database Syst Rev. 2016;11:CD011227.

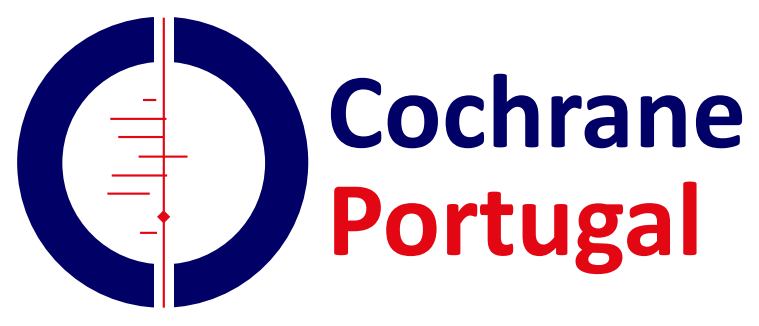

